\title{
A Flat Manifold with No Symmetries
}

\author{
Reinhard Waldmüller
}

\section{CONTENTS}

1. Introduction

2. The Example

3. Lattices and Cocycles

Acknowledgments

References

2000 AMS Subject Classification: Primary 20C34, 20H15, 53C25

Keywords: Flat manifolds, Bieberbach groups
In this note, we give an example of a flat manifold having a trivial group of affinities by constructing a Bieberbach group with a trivial center and trivial outer automorphism group.

\section{INTRODUCTION}

The compact, connected, flat Riemannian manifolds (flat manifolds for short) are classified up to affine equivalence by their fundamental groups, the so-called Bieberbach groups. These groups are precisely the torsion-free groups satisfying an exact sequence

$$
0 \longrightarrow L \longrightarrow \Gamma \longrightarrow G \longrightarrow 1
$$

where $G$ is a finite group and $L$ is a faithful $\mathbb{Z} G$-lattice of finite rank, i.e., a free $\mathbb{Z}$-module of finite rank on which $G$ acts faithfully. Let $X$ be a flat manifold with fundamental group $\Gamma$. The group $\operatorname{Aff}(X)$ of affine self-equivalences of $X$ is a Lie group. Its identity component $\operatorname{Aff}_{0}(X)$ is a torus whose dimension is the rank of the center of $\Gamma$, and $\operatorname{Aff}(X) / \operatorname{Aff}_{0}(X)$ is isomorphic to $\operatorname{Out}(\Gamma)$, the outer automorphism group of $\Gamma$. Malfait conjectured [Malfait 98, Conjecture 5.13], that $\operatorname{Aff}(X)$ is never torsion-free (where the trivial group is considered to be torsion-free). In Section 2, we will give an example of a Bieberbach group that has a trivial center and trivial outer automorphism group, and hence is the fundamental group of a flat manifold with trivial group of affinities. In particular, it is a counterexample to Malfait's conjecture. Let $\Gamma$ be a Bieberbach group as in $(1-1)$ and $\delta \in H^{2}(G, L)$ be the cohomology class giving rise to $(1-1)$. Let $N$ be the normalizer of $G$ in $\operatorname{Aut}(L)$. There is a natural action of $N$ on $H^{2}(G, L)$, and $\operatorname{Out}(\Gamma)$ satisfies the short exact sequence (see [Charlap 86, Theorem V.1.1])

$$
0 \longrightarrow H^{1}(G, L) \longrightarrow \operatorname{Out}(\Gamma) \longrightarrow N_{\delta} / G \longrightarrow 1,
$$

where $N_{\delta}$ denotes the stabilizer of $\delta$ in $N$. The center of $\Gamma$ is $L^{G}=\{v \in L \mid g v=v \forall g \in G\}$, so to find a flat manifold with no symmetries, it suffices to construct a 
FIGURE 1. $L_{2}$. 


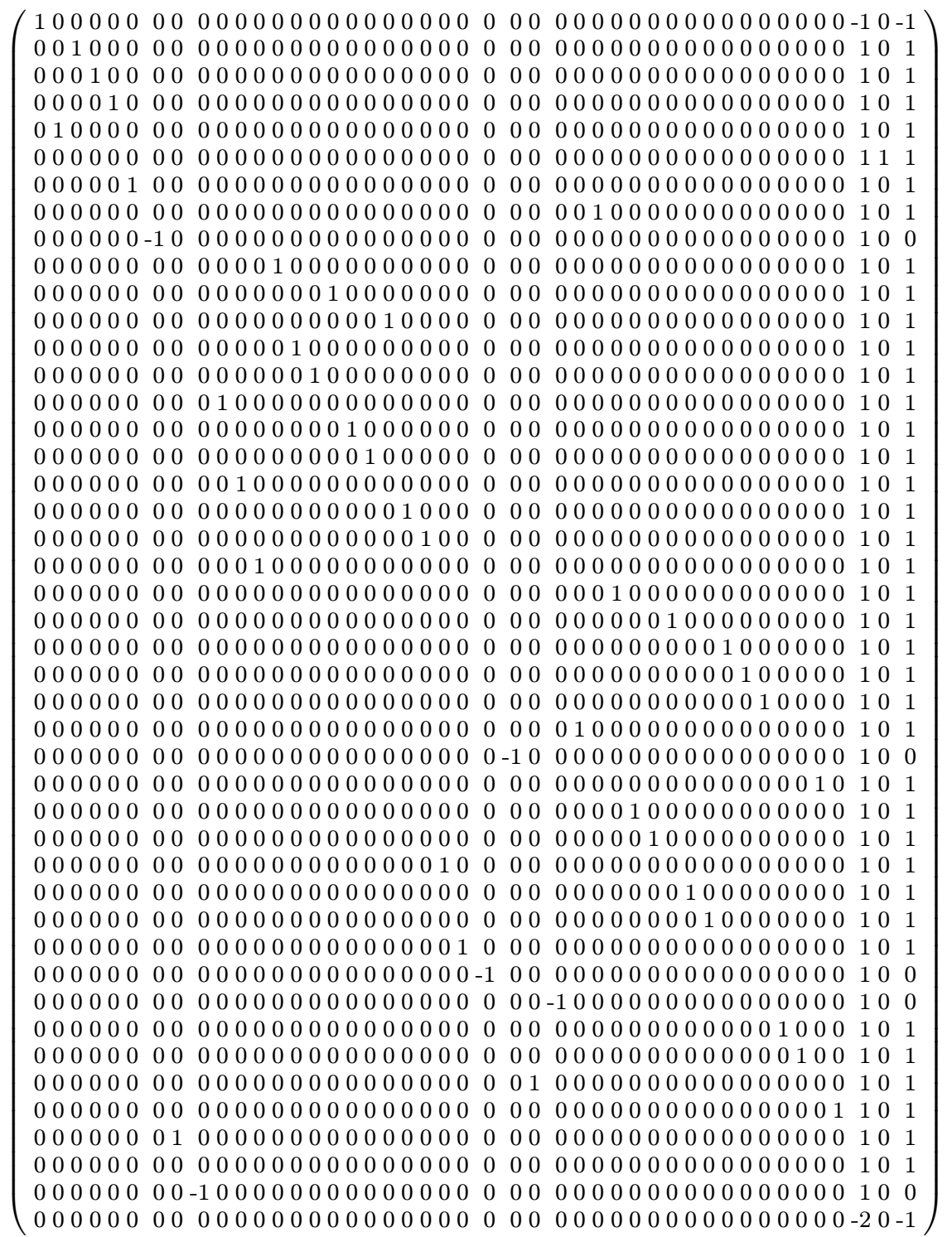

Figure 2(b). 


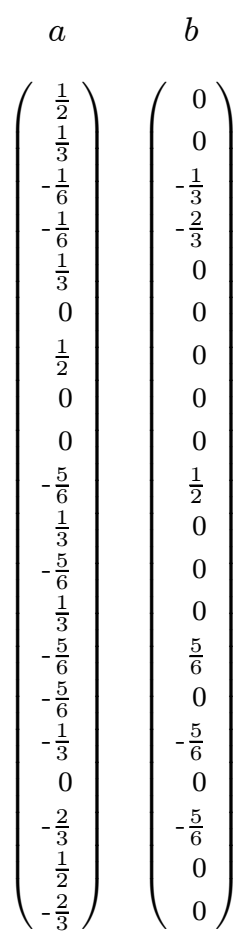

$\delta_{1}$

\begin{tabular}{|c|c|}
\hline$a$ & $b$ \\
\hline$\left.\frac{4}{5}\right)$ & $\left(-\frac{4}{5}\right)$ \\
\hline 0 & $\frac{2}{5}$ \\
\hline 0 & $\frac{2}{5}$ \\
\hline$-\frac{1}{5}$ & 0 \\
\hline$\frac{2}{5}$ & $-\frac{3}{5}$ \\
\hline$\frac{2}{5}$ & $\begin{array}{l}\frac{1}{5} \\
1\end{array}$ \\
\hline 0 & $-\frac{1}{5}$ \\
\hline$\frac{2}{5}$ & 0 \\
\hline 0 & 0 \\
\hline 0 & 0 \\
\hline$-\frac{2}{5}$ & 0 \\
\hline$-\frac{4}{5}$ & 0 \\
\hline$-\frac{2}{5}$ & 0 \\
\hline$\frac{2}{5}$ & 0 \\
\hline$\frac{2}{5}$ & 0 \\
\hline$-\frac{4}{5}$ & 0 \\
\hline$\frac{3}{5}$ & 0 \\
\hline$-\frac{1}{5}$ & 0 \\
\hline$-\frac{2}{5}$ & 0 \\
\hline$-\frac{2}{5}$ & 0 \\
\hline$-\frac{1}{5}$ & 0 \\
\hline$-\frac{4}{5}$ & 0 \\
\hline$\frac{1}{5}$ & 0 \\
\hline 0 & 0 \\
\hline 0 & 0 \\
\hline$\frac{2}{5}$ & 0 \\
\hline$\frac{2}{5}$ & 0 \\
\hline 0 & 0 \\
\hline$-\frac{3}{5}$ & 0 \\
\hline \begin{tabular}{l|}
$-\frac{4}{5}$ \\
-1
\end{tabular} & $\begin{array}{l}0 \\
0\end{array}$ \\
\hline & $\left.\frac{1}{5}\right)$ \\
\hline
\end{tabular}

$\delta_{2}$

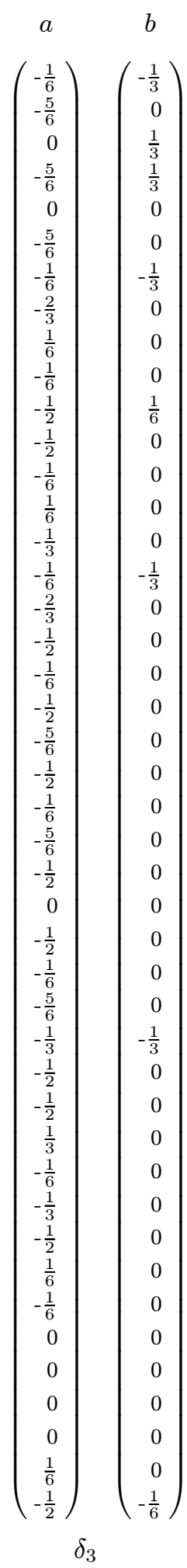

FIGURE 3. The cocycles.

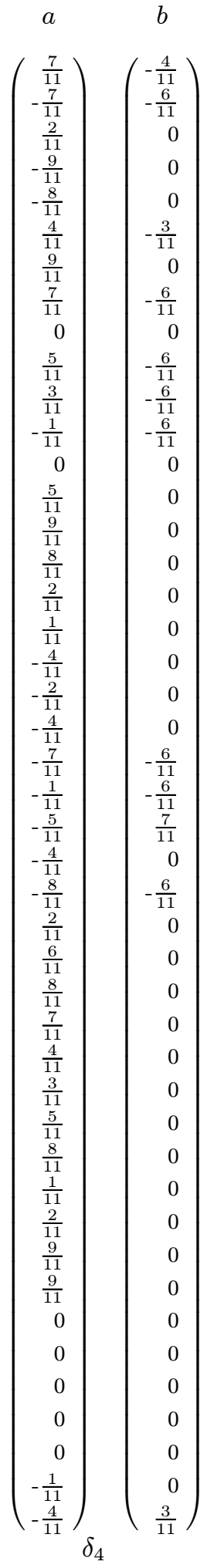


torsion-free extension (1-1) such that $L^{G}, H^{1}(G, L)$, and $N_{\delta} / G$ are trivial. To do this, we need to know when an extension (1-1) is torsion-free, and this happens if and only if we have $\operatorname{res}_{U}^{G}(\delta) \neq 0$ for all nontrivial subgroups $U$ of $G$, where $\operatorname{res}_{U}^{G}: H^{2}(G, L) \rightarrow H^{2}(U, L)$ denotes the restriction homomorphism (see [Charlap 86, Theorem III.2.1]). An element $\delta \in H^{2}(G, L)$ satisfying this condition is called special. By transitivity of restriction, it suffices to check this for subgroups of prime order, and by the action of $G$ on $H^{2}(G, L)$, it suffices to consider representatives of conjugacy classes of subgroups. Also, for a Sylow $p$-subgroup $U$ of $G$, the restriction homomorphism $\operatorname{res}_{U}^{G}: H^{2}(G, L)_{p} \rightarrow H^{2}(U, L)$ is injective. Since it is difficult to compute $H^{2}(G, L)$, we use the isomorphic group $H^{1}\left(G, \mathbb{Q} \otimes_{\mathbb{Z}} L / L\right)$ instead.

\section{THE EXAMPLE}

Let $G=M_{11}$, the Mathieu group on 11 letters. Then $G$ has a presentation

$$
\begin{array}{r}
G \cong\langle a, b| a^{2}, b^{4},(a b)^{11},\left(a b^{2}\right)^{6}, \\
\left.a b a b a b^{-1} a b a b^{2} a b^{-1} a b a b^{-1} a b^{-1}\right\rangle,
\end{array}
$$

and representatives of conjugacy classes of subgroups of order 2, respectively, 3 are $\langle a\rangle$, respectively, $\left\langle\left(a b^{2}\right)^{2}\right\rangle$; see [Wilson et al. 01]. Let $L_{1}$ be the 20-dimensional integral representation of $G$ from the Web-Atlas [Wilson et al. 01], let $L_{3}$ be the dual of the 44-dimensional integral representation of $G$ from the Web-Atlas, and let $L_{2}$ and $L_{4}$ be the lattices given in Figure 1 and Figure 2 , respectively. The lattices are given by the images of the generators $a$ and $b$ under the corresponding integral representation of $G$, i.e., the lattice is identified with $\mathbb{Z}^{n}$ on which $G$ acts by matrix multiplication. Furthermore, let $\delta_{i} \in H^{1}\left(G, \mathbb{Q} \otimes_{\mathbb{Z}} L_{i} / L_{i}\right)$ for $1 \leq i \leq 4$ be the cocycles given in Figure 3. A cocycle $\delta$ is given by vectors $v_{a}, v_{b} \in \mathbb{Q}^{n}$ such that $\delta(a)=v_{a}+\mathbb{Z}^{n}$ and $\delta(b)=v_{b}+\mathbb{Z}^{n}$. These have the following properties:

(1) The character afforded by $L_{1}$ is $\chi+\bar{\chi}$, where $\chi$ is one of the two nonreal irreducible characters of $G$ of degree 10. The order of $\delta_{1}$ is 6 , and we have $\operatorname{res}_{\langle a\rangle}^{G}\left(\delta_{1}\right)=0$, but $\operatorname{res}_{\left\langle\left(a b^{2}\right)^{2}\right\rangle}^{G}\left(\delta_{1}\right) \neq 0$.

(2) The character afforded by $L_{2}$ is $\chi+\bar{\chi}$, where $\chi$ is one of the two irreducible characters of $G$ of degree 16. The order of $\delta_{2}$ is 5 . Hence the restriction of $\delta_{2}$ to any subgroup of order 5 is nonzero.

(3) The character afforded by $L_{3}$ is the irreducible character of $G$ of degree 44 . The order of $\delta_{3}$ is 6 , and we have $\operatorname{res}_{\langle a\rangle}^{G}\left(\delta_{3}\right) \neq 0$, but $\operatorname{res}_{\left\langle\left(a b^{2}\right)^{2}\right\rangle}^{G}\left(\delta_{3}\right)=0$.
(4) The character afforded by $L_{4}$ is the irreducible character of $G$ of degree 45 . The order of $\delta_{4}$ is 11 . Hence the restriction of $\delta_{4}$ to any subgroup of order 11 is nonzero.

Thus $\delta:=\delta_{1}+\cdots+\delta_{4} \in H^{1}\left(G, \mathbb{Q} \otimes_{\mathbb{Z}} L / L\right)$, where $L:=L_{1} \oplus \cdots \oplus L_{4}$, is a special element. Let $\Gamma$ be an extension of $L$ by $G$ given by $\delta$. Then $\Gamma$ is torsion-free and has trivial center. Moreover, we have $H^{1}(G, L)=0$. This is easily checked using the fact that, if $L^{G}=0$, a prime $p$ divides $\left|H^{1}(G, L)\right|$ if and only if $(L / p L)^{G} \neq 0$ (see [Hiss and Szczepański 97, Lemma 2.1]). Now it remains to check that $N_{\operatorname{Aut}(L)}(G)_{\delta}=G$. Since $G$ has no outer automorphisms, we have $N_{\operatorname{Aut}(L)}(G)=C_{\operatorname{Aut}(L)}(G) G$, and the centralizer of $G$ in $\operatorname{Aut}(L)$ is $C_{\mathrm{Aut}\left(L_{1}\right)}(G) \times \cdots \times$ $C_{\operatorname{Aut}\left(L_{4}\right)}(G)$. We claim that $C_{\operatorname{Aut}\left(L_{i}\right)}(G)=\{ \pm 1\}$ for $1 \leq i \leq 4$. This is obvious for $i=3,4$. Now $C_{\mathrm{Aut}\left(L_{i}\right)}(G)$ is the unit group of $\operatorname{End}_{\mathbb{Z} G}\left(L_{i}\right)$, which is a $\mathbb{Z}$-order in $\operatorname{End}_{\mathbb{Q} G}\left(\mathbb{Q} \otimes_{\mathbb{Z}} L_{i}\right)$. For $i=1,2$, this endomorphism ring is isomorphic to $\mathbb{Q}(\chi)$, where $\chi$ is as above. In the first case, $\mathbb{Q}(\chi)=\mathbb{Q}(\sqrt{-2})$, and in the second case, we have $\mathbb{Q}(\chi)=\mathbb{Q}(\sqrt{-11})$. In both cases, all $\mathbb{Z}$-orders have unit group $\{ \pm 1\}$, hence the claim. Now it is clear that $C_{\mathrm{Aut}(L)}(G)_{\delta}=1$ and we are done.

The computations in this example have been performed with GAP [GAP 02] and CARAT [Opgenorth et al. 01].

It would be desirable to have more than one example, preferably an infinite family. But to achieve this using the above strategy, one needs a family of lattices with the "right" properties, and I do not know of such a family.

\section{ACKNOWLEDGMENTS}

This example is the main result of my diploma thesis and I am grateful to my advisor G. Hiss for helpful hints and his encouragement to publish it. Also, I would like to thank J. Müller for several discussions.

\section{REFERENCES}

[Opgenorth et al. 01] J. Opgenorth, W. Plesken and T. Schulz. "CARAT - Crystallographic Algorithms and Tables," Version 1.2, 2001. Available from World Wide Web (http://wwwb.math.rwth-aachen.de/carat/), 2001.

[Charlap 86] L. S. Charlap. Bieberbach Groups and Flat Manifolds. New York: Springer-Verlag, 1986.

[GAP 02] The GAP Group. "GAP - Groups, Algorithms, and Programming," Version 4.3, 2002. Available from World Wide Web (http://www.gap-system.org), 2002. 
[Hiss and Szczepański 97] G. Hiss and A. Szczepański. "Flat Manifolds with Only Finitely Many Affinities." Bull. Polish Acad. Sci. Math. 45:4 (1997), 349-357.

[Malfait 98] W. Malfait."Model Aspherical Manifolds with No Periodic Maps." Trans. A.M.S. 350:11 (1998), 46934708.

Received January 23, 2003; accepted April 29, 2003.

\footnotetext{
Reinhard Waldmüller, Lehrstuhl D für Mathematik, RWTH Aachen, Templergraben 64, 52062 Aachen, Germany (reinhard@math.rwth-aachen.de)
}

[Wilson et al. 01] R. A. Wilson, P. Walsh, J. Tripp, I. Suleiman, S. Rogers, R. A. Parker, S. Norton, S. Linton, and J. Bray. "Atlas of Finite Group Representations." Online database. Available from World Wide Web (http://www.mat.bham.ac.uk/atlas/), 2001. 